\title{
LAS FRONTERAS NO ADYACENTES, COMO SOLUCIÓN AL TRÁFICO AÉREO ILEGAL
}

\author{
Brigadier General HENRY QUINTERO BARRIOS ${ }^{l}$
}

El propósito de este documento es interpretar qué se puede entender por fronteras no adyacentes y evidenciar cómo están presentes en el fenómeno del movimiento aéreo ilegal, y la forma en que se ha logrado controlar esta actividad por parte de la Fuerza Aérea Colombiana, por medio de diferentes estrategias que involucran a otras entidades, agencias, organizaciones y países, a través de la metodología de seis sigma, y su impacto en el tema fronterizo, entre el año 2003 y 2013, como un programa efectivo.

En primer término es necesario enfocarse en qué se entiende por frontera, y su planteamiento desde diferentes perspectivas, tanto históricas, como realistas o ideológicas y culturales con el fin de poder entender el desarrollo de esta área y cómo impacta el devenir de una nación, así mismo el origen, no solamente del término mismo, sino de su realidad geográfica y cuál es el papel que cumple en la actualidad.

La definición de frontera tal como se le conoce hoy en día ha sido una evolución que viene desde la antigüedad, aunque el término convencional de demarcación de un país con respecto a otro en la antigüedad partía de la consideración del propio país como centro de poder y civilización y al resto se le consideraban pueblos bárbaros, obviamente desde la óptica de superioridad cultural, política y militar del país en cuestión, y desde entonces se denomina límite (o marca) a la localización geográfica de "tierra de nadie" u "otra tierra", que separa dos realidades, con una connotación política sobre una realidad geográfica contrastable. Tanto en la antigüedad, como en el medioevo (FERNÁNDEZ-CARrión, 2010, p. 3), con diferentes conceptualizaciones y en muchos casos adaptada a las necesidades de los gobernantes de turno.

Inicialmente, hay que considerar las diferencias entre frontera y límite, y etimológicamente Julio Casares en el diccionario ideológico de la lengua española indica que por límite, se entiende el término o lindero de reinos, posesiones, etc.,

Jefe de la Jefatura Tecnologías de la Información JETIC. Administrador Aeronáutico, Magíster en Estrategia y Geopolítica de la Escuela Superior de Guerra; especialista en telecomunicaciones. 
e incluso figuradamente como el fin, o término. Mientras que, frontera, es el límite o confín de un Estado (CASARES, J., 1995), dentro de este contexto se ve como una definición se ajusta a un significado geográfico, mientras la otra hace referencia a un contexto político.

Por otra parte, es Frederick Jackson Turner quien inventa el término frontera, en 1893, en "El significado de la frontera en la historia americana", "la frontera es el borde exterior de la ola, el punto de contacto entre la barbarie y la civilización" (TURNER, 1893, p. 188), y la hace sinónima del espíritu nacional norteamericano. Posteriormente, Michel Foucher en L'Invention des frontières, se cuestiona la frontera desde una doble perspectiva, como estrategia política interna o nacional o como poder geopolítico a nivel internacional (1987).

En la actualidad el término frontera reúne la concepción geográfica y política, cultural, étnica, económica, etc. que existe entre dos o más países. Por esto, se puede hablar de frontera nacional, regional, continental, religiosa hasta personal, de un individuo o colectivo con respecto al conjunto del que se pretende diferenciar. Por ello, la frontera puede hacer mención a un colectivo, como a un individuo, a un país como a una región, a una localización geográfica, como una realidad política hasta una diferenciación cultural, religiosa o económica. Aunque, normalmente predominará la concepción de frontera como sinónima de una realidad política, localizada dentro de unas coordenadas geográficas determinadas por encima del resto de las demás consideraciones etimológicas del término indicadas. (FERNÁNDEZ-CARRIón, 2010, p. 4).

En 1980, Jean Gottmann, se cuestiona la frontera indistintamente como línea o zona ("la frontière est une ligne ou une zone"), mientras que, ese mismo año, John House entiende como frontera ('frontier') la zona que se extiende a los dos lados de una línea que marca una frontera ('boundary')...En este mismo sentido, Michael Foucher en L'Invention des frontières resalta que por una cuestión semántica, y añadiré personalmente que política, a partir de Turner, los norteamericanos consideran la frontera como la conjunción de "the frontier" (frontera) y "boundary" (límite), y por ello el autor defiende la diferenciación lingüística propuesta por otros investigadores franceses que consideran como "frontières" el término geopolítico por excelencia, en el que se diferencia entre "ligne et zone correspondent" (entre línea y zona de frontera). (FouCHER, 1987, p. 38), así se plantea la diferencia de manera expresa entre límite y zona de frontera, una concepción geográfica y una política.

Para el caso de Hispanoamérica existe una unidad política, jurídica, económica, social, religiosa, lingüística, etc. que es establecida por la corona castellana, como lo expresa Alfredo Jiménez Núñez en "The Spanish Colonial Model'. ( 1994, pp. 66-95). 
En el libro de Historiografía metodología y tipología de fronteras, se hace una diferenciación desde varios puntos de vista de las fronteras, y expresada inicialmente desde la perspectiva del tiempo y el espacio.

Así mismo, plantea como a través de la historia las fronteras se han definido de manera preferencial por las referencias geográficas, con lo cual establece las fronteras naturales y por concomitancia las fronteras artificiales como herencia del proceso de descolonización principalmente.

Por otra parte, la combinación de las dos categorías anteriores da origen a las fronteras mixtas, que en la actualidad se pueden ver a lo largo de frontera colombo venezolana, y que por sustracción de materia son preponderantes en la definición de límites marítimos.

Igualmente, se establece una subdivisión en las fronteras naturales referenciando fronteras territoriales e insulares, donde las insulares por antonomasia definen una relación de los países insulares con su entorno debido a la falta de relación directa y de contacto visual y físico con el país vecino.

El Doctor Miguel Héctor Fernández Carrión Director del Instituto de Estudios Históricos y Económicos en Madrid España, autor del mismo libro plantea otra diferenciación entre fronteras urbanas y rurales, pues en general en las fronteras hay espacios vacíos que se pueden definir como rurales y las que hacen referencia a centros poblados donde conviven las comunidades de dos o más Estados, como es el caso de Leticia (Colombia), Tabatinga (Brasil) y Santa Rosa (Perú), y que en la actualidad tienen libre tránsito de bienes y personas.

Por otra parte, Hartshorne en the nature of geography, entiende la existencia de "límites políticos", pero al mismo tiempo considera la formación de las fronteras políticas en relación con los procesos de poblamiento, por lo que distingue seis tipos o etapas: "fronteras totalmente anteriores o pioneras; anteriores; subsiguientes (...); sobrepuestas (...); sobrepuesta de modo excavación y reliquias (...)”(1939).

Sin embargo, las fronteras no adyacentes no se han expuesto y se plantea dicha definición como las líneas de comunicación directas que permiten el intercambio de bienes y servicios que impactan en los intereses nacionales sin la intermediación de terceros estados.

Partiendo de esta definición, se puede ver que existen acuerdos, tratados, convenios que permiten el intercambio de mercancías, personas, bienes, tanto de manera directa en forma legal, como es el caso de exportaciones, importaciones, que a su vez establecen rutas que permiten el actuar de las organizaciones que operan cometiendo delitos transnacionales y establecen a su vez líneas directas de comunicación para el tráfico de drogas, armas, trata de personas, migración ilegal, dumping, lavado de activos, etc. 
En el primer caso ingresan categorías que van desde el turismo, con la promoción de los diferentes atractivos de cada país, con excepciones de requisitos legales, y barreras comerciales, hasta el intercambio de bienes y mercancías con aranceles preferenciales, reducción de trámites y facilidades logísticas y legales que incentivan dicho intercambio, adicionalmente, se encuentran los servicios que no necesariamente requieren una presencia física en los Estados no adyacentes, sino que pueden ser provistos de manera remota y para lo cual es necesario establecer canales que permitan dicha actividad, todas estas conllevan a su reconocimiento en temas como tributación, cumplimiento de requisitos y normatividad, respeto del entorno cultural y social, idioma, costumbres etc., para ajustarse a las necesidades particulares de dicho Estado no adyacente y de las preferencias de sus habitantes con el fin de garantizar el poder competir en dichos mercados, pero desde la perspectiva política también se puede observar como un estado no adyacente es limítrofe con otro. El solo hecho de establecer una misión diplomática recorta los canales comunicacionales y de relaciones con este estado, de tal suerte que se pueden adelantar en dicha misión tareas que de otra forma involucrarían o a terceros estados o requerirían un esfuerzo adicional, así mismo la presencia de actores diplomáticos permite la participación directa de sus conciudadanos en la actividad electoral como una extensión de los derechos de los connacionales y no solamente en lo político, también en lo administrativo y en lo económico con lo cual se establecen relaciones que naturalmente están circunscritas a países adyacentes, validando nuevamente que no es geográficamente necesario ser países vecinos para tener una frontera común.

Por otra parte, desde la perspectiva geopolítica cabe anotar la relación entre estados que define Kautilya en Arthasastra, los estados vecinos son los enemigos naturales, mientras que los estados no colindantes son los aliados naturales, basado en que dicha premisa igualmente aplica para los estados vecinos quienes por su naturaleza tendrán como enemigos naturales a dichos estados y a terceros estados no colindantes como aliados naturales.

En lo referente al movimiento ilegal aéreo en Colombia, este se inició con la bonanza marimbera en la Guajira Colombiana en los años 70, fenómeno que fue migrando hacia la producción de narcóticos, y desde la producción de hoja de coca inicialmente en los 80 y en los 90 a la producción de pasta base de coca y Clorhidrato de cocaína, aumentando exponencialmente las utilidades de los grupos al margen de la ley, aprovechando que las facilidades que genera el movimiento aéreo y las debilidades existentes para el control del mismo por parte de las autoridades (KaUtiLYA, 2008).

"Los delincuentes dedicados al negocio del narcotráfico han diseñado diferentes estrategias para lograr movilizar sus mercancías a los puntos estratégicos de distribución y comercialización, logrando así continuar con la cadena de esta estructura criminal; el medio más usado en la década de los 80's (inicios del 
auge del narcotráfico) fue el terrestre, pero al ser el más controlado por la fuerza pública en esa época, tener la limitante de cobertura internacional y el tiempo excesivo de movilización, los narcotraficantes incursionaron en los medios marítimo y aéreo para efectuar sus movimientos ilegales, siendo este último el más rápido y de mayor cobertura. En consecuencia nace la preocupación de realizar un estudio de los movimientos aéreos ilegales concentrados en la región Caribe" (Devia, Iván, \& Ferreira Cardenas, 2015, p. 3).

En Colombia la administración del espacio Aéreo nacional está regida por la Unidad Administrativa Especial de Aeronáutica Civil (UAEAC), quien se encarga de hacer cumplir los convenios establecidos con la Organización de Aviación Civil Internacional (OACI), que es un organismo creado por la Organización de Naciones Unidas (ONU) en 1944, y cuya misión es "En la Aerocivil trabajamos por el desarrollo ordenado de la aviación civil, de la industria aérea y la utilización segura del espacio aéreo colombiano, facilitando el transporte intermodal y contribuyendo al mejoramiento de la competitividad del país.", que en la práctica se traduce en la gestión del espacio aéreo para que las aeronaves legales vuelen con seguridad.

Por otra parte, "La Fuerza Aérea Colombiana ejerce y mantiene el dominio del espacio aéreo, conduce operaciones aéreas, para la defensa de la soberanía, la independencia, la integridad del territorio nacional, el orden constitucional y el logro de los fines del Estado." como se puede ver es la Fuerza Aérea Colombiana la encargada de mantener bajo control que no se efectúen vuelos ilegales en el Espacio Aéreo Nacional.

Partiendo de aquí, se inicia la lucha contra el narcotráfico por vía aérea como una necesidad del gobierno Colombiano a través de la Fuerza Aérea Colombiana y se establecen las primeras actividades en 1990 con el apoyo frontal del gobierno de los EEUU, iniciando los sobrevuelos de aeronaves AWAC de la USAF, P-3B y C-550 de USCUSTOMS, con operadores de Defensa Aérea Colombianos, Peruanos y Ecuatorianos abordo, en un esfuerzo por lograr una sinergia en la interdicción de aeronaves y embarcaciones que cruzaran el Gran Caribe con rumbo norte, sin embargo dicho esfuerzo estaba muy por debajo de las necesidades de vigilancia y monitoreo de dicha zona, como se puede ver con esta simple iniciativa los intereses del Gobierno de los EEUU de mantener su territorio libre del flagelo de las drogas, lo lleva a trabajar con países limítrofes no colindantes para establecer una acción directa sobre una actividad que afecta su seguridad y en la cual no intervenían terceros estados que podían servir de área de amortiguación para tratar de controlar la entrada de narcóticos, pues la práctica de la época era el arrojar los cargamentos de droga al mar o en zonas despobladas del territorio para ser recuperadas por las organizaciones al margen de la ley.

Por lo anterior, en 1987 se inicia el programa Caribean Basin Radar Network (CBRN), que tenía como objetivo establecer una red de radares que cubriera una zona desde los países desde donde salían los vuelos ilegales cargados con droga y se implementan radares en Colombia en Puerto Salgar en 1989, Villavicencio 
y Barranquilla en 1990, los cuales iniciaron como equipos tácticos móviles, TPS 43 y TPS 47, en donde todo el trabajo logístico, de soporte y de mantenimiento estaba a cargo de los EEUU y la operación de vigilancia y seguimiento a cargo de personal de las fuerzas aéreas de los países anfitriones, una vez más dejando entrever la frontera existente entre países no colindantes, con intereses comunes desde dos ópticas diferentes, por una parte los EEUU interesados en que no ingresaran narcóticos a su país y por otra parte los países productores tratando de reducir y someter a las organizaciones al margen de la ley que afectan la seguridad y convivencia de sus ciudadanos.

En 1991 se implementa la operación andina "Plan Águila", cuyo propósito primordial era el control de vuelos ilegales desde Ecuador, Perú y el sur de Colombia, áreas de producción y procesamiento de pasta de base de coca, por lo tanto se instalan radares en Colombia en Tres Esquinas, Larandia y Araracuara (Caquetá), Leticia (Amazonas), Iquitos (Perú) y Lago Agrio (Ecuador), igualmente con el apoyo de los EEUU y cuya señal era explotada por cada país y los EEUU, quienes tenían interés en efectuar la interdicción de los vuelos ilegales, con lo cual se deja ver otro aspecto de las fronteras no adyacentes, pero de intereses comunes.

Por otra parte, está la situación de vuelos ilegales en Colombia para el año 2003 "En la Figura 1, se observa el número de vuelos ilegales despegando y aterrizando en territorio colombiano en el año 2003. Se registró un promedio de casi dos vuelos diarios (para un total de 639) en los cuales traficaban estupefacientes movilizándolos a los países fronterizos, convirtiendo el fenómeno en un crimen transnacional." (DEviA et al., 2015, p. 5),

Figura 1. Trazas de vuelos ilegales en Colombia en el año 2003

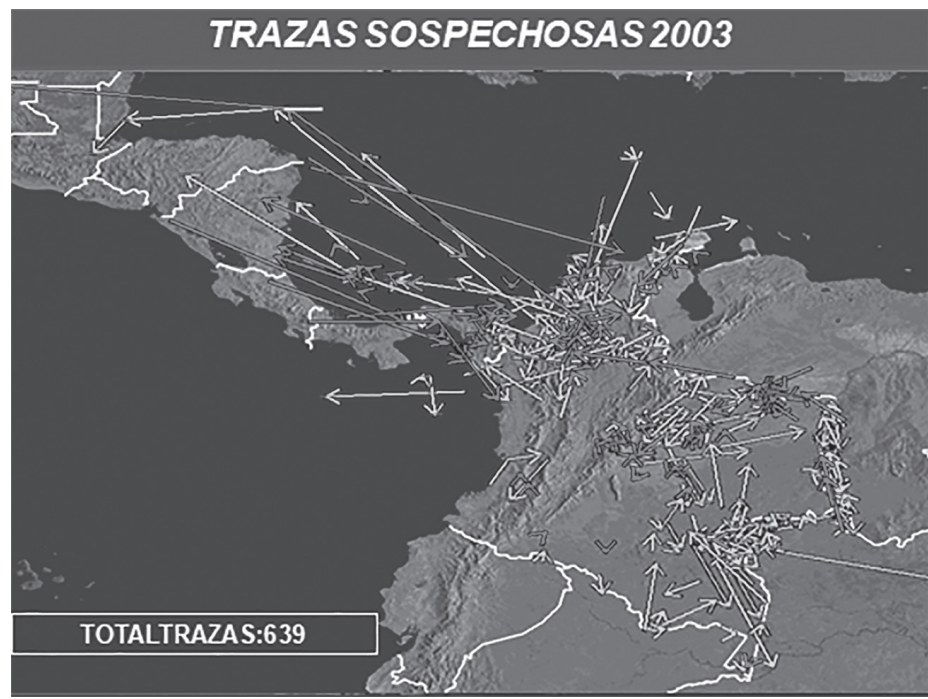

Fuente: Dirección Defensa Aérea de la FAC. 
Adicionalmente, si se considera que cada vuelo tenía en promedio una capacidad de carga de una Tonelada métrica de clorhidrato de cocaína (HCL), se puede inferir que se transportaron 639 toneladas durante el año 2003, por vía aérea solamente, y el valor del kilo de HCL en las calles de los EEUU era de USD 25.000,oo, se puede concluir que por el tráfico de HCL por esta vía las organizaciones al margen de la ley recibieron USD 15.975.000.000,oo, sin considerar que cada kilo de HCL puede convertirse hasta en 4 kilos con la adición de otros productos, como harinas, cementos, "La cocaína, entonces se vende en las calles como hidrocloruro de cocaína, no apta para fumar, o como formas intermedias menos costosas, como el crack o el bazuco, aptas para fumar. Los vendedores en las calles la diluyen para reducir sus costes, con sustancias inertes de apariencia similar como talco, azúcar, suero, o con drogas activas como procaína y benzocaína u otros estimulantes del sistema nervioso como anfetaminas. La pureza de la cocaína es variable" (GONZÁLEZ \& VilLASENÍN, 2010, p. 5).

Como se puede ver, los ingresos de los carteles son cuantiosos y les permiten a través de las mismas rutas mover hacia los EEUU, narcóticos, pero en sentido contrario armas y dinero, con lo cual el ciclo comercial para estas organizaciones era completo, según el banco mundial para el año 2003 el PIB de Colombia correspondía a USD 94.680.000.000, con lo cual se puede observar que las organizaciones al margen de la ley recibieron el 16.87\% del PIB de Colombia, lo que fácilmente coloca en riesgo la capacidad del Estado, especialmente en lo referente a su legitimidad y actuación en la sociedad, ya que estas organizaciones terminan llenando los espacios que los entes gubernamentales no son capaces de llenar y el mismo estado reconoce a través de sus entidades que es un problema, como lo expresa el Departamento Administrativo Nacional de Estadísticas (DANE) en su boletín del cuarto trimestre del año 2003 "El PIB sin cultivos ilícitos registró una tasa de crecimiento de 3,96\%"(DANE, 2003, p. 1).

"Las nuevas rutas movilizan las drogas ilícitas desde territorios aledaños a las fronteras colombianas hacia el Caribe, afectando el territorio Nacional de los países fronterizos, sin embargo las aeronaves que realizan esa actividad logran sobrevolar parte del espacio aéreo colombiano, al cruzar el Mar Caribe, atravesando parte del mar patrimonial ${ }^{2}$, Dado que por normas internacionales no se puede hacer uso de las armas en aguas patrimoniales ${ }^{3}$, el Estado colombiano soporta su lucha contra el Narcotráfico con la implementación de convenios ${ }^{4}$ para combatir este delito de manera conjunta con los demás países de la región que se ven afectados por este delito.'(Devia et al., 2015, p. 6).

Franja de mar desde el mar territorial hasta las 200 Millas Náuticas.

Franja de mar que va más allá del mar territorial hasta el límite de las aguas jurisdiccionales.

Procedimientos operacionales Vigentes. Fuerza Aérea Colombiana en convenios con EE.UU, Honduras,

República Dominicana, Guatemala, Perú, Brasil, Venezuela, Ecuador y Panamá. 
El profesor Rubén Darío López en su trabajo sobre mar territorial y mar patrimonial define como:

"Mar patrimonial

1. El Estado ribereño tiene el deber de promover y el derecho de reglamentar las investigaciones científicas que se adelantan en el mar patrimonial, así como el de aportar las medidas necesarias para evitar la contaminación del medio marino y asegurar su soberanía sobre los recursos.

2. El Estado ribereño ejerce derechos de soberanía sobre los recursos tanto renovables como no renovables que se encuentran en las aguas, en el lecho y el subsuelo de una zona adyacente al mar territorial, denominada mar patrimonial.

3. La anchura del mar patrimonial debe ser objeto de acuerdo internacional preferentemente de ámbito mundial. La suma de esta zona y la del mar territorial, teniendo en cuenta las circunstancias geográficas, no deberá exceder en total de 200 millas náuticas.

4. La delimitación de esta zona entre dos o más estados se hará con arreglo a los procedimientos pacíficos previstos en la Carta de las Naciones Unidas.

5. En el mar patrimonial, las naves y aeronaves de todos los Estados con litoral marítimo o sin él, tienen el derecho de libre navegación y sobrevuelo sin otras restricciones que las que pueden resultar del ejercicio, por parte del estado ribereño, de sus derechos en el mismo mar. Con estas únicas limitaciones, habrá también libertad para tender cables y tuberías submarinas.'(López, 1981, p. 154).

Con lo anterior quedan perfectamente claro los derechos del Estado a ser ejercidos en su mar territorial y mar patrimonial, así mismo, como se puede observar en la figura 1, estas rutas cruzan en algún momento el mar territorial y el mar patrimonial, de por lo menos tres estados antes de llegar a su destino, varios de ellos no adyacentes, pero limítrofes en lo que se refiere a esta vulnerabilidad y al interés común de interdictar dichos vuelos, de este interés nace la necesidad de establecer acuerdos como los Procedimientos Vigentes Operacionales (POV ), que se han firmado con varios países de la región, donde se estandarizan los procedimientos a ejecutar cuando se tiene una aeronave ilegal cruzando el espacio aéreo de un Estado hacia otro sin cumplir con los requisitos establecidos por la OACI, con lo cual se generan dos riesgos, el primero, en lo que hace referencia al tráfico de drogas, un delito transnacional, y el segundo, al que se genera en contra de la aviación comercial que está volando sin saber de la posición y altura de dichas aeronaves que pueden llegar a confluir con el tráfico ilegal sin preservar ni las distancias o alturas mínimas de seguridad.

En lo que respecta a Colombia, se definieron los procedimientos de interdicción de aeronaves con el gobierno de los EEUU en agreement between the government 
of the united states of america and the government of the republic of Colombia concerning the program for the suppression of illicit aerial traffic in narcotic drugs and psychotropic substances ("air bridge denial agreement") del 2004 y actualizado en el 2010, basados en el documento de naciones unidas, uso de las armas por las autoridades encargadas de hacer cumplir la ley, con el fin de garantizar que se preservara el respeto de los Derechos Humanos, así mismo para avalar su cumplimiento se encuentra un programa de entrenamiento para las tripulaciones de la FAC, con el fin de certificar su estandarización, de otra parte no solamente siguen el procedimiento quienes se encuentran en vuelo, sino que el mismo es monitoreado, seguido y aprobado por un oficial supervisor en tierra, quien escucha el procedimiento y aprueba sus pasos sucedáneos, y de forma similar otro supervisor en tierra en Joint Interagency Task Force of South (JIATF of South), con el fin de atestiguar el respeto de los procedimientos y de los Derechos Humanos, así mismo, el Programa Air Bridge Denied (ABD), es sometido a varias etapas de control, en primera instancia reuniones periódicas durante el año con JIATF of south para verificación de los eventos transcurridos entre cada revisión, en segunda instancia la evaluación anual por parte del congreso de los EEUU y autorizado previa revisión, por el presidente de los EEUU, lo que en la práctica se convierte en un garante de la correcta aplicación de procedimientos, alto nivel de entrenamiento y respeto de los Derechos Humanos.

En todo el proceso y estas revisiones se tienen en cuenta el respeto de los DDHH bajo la premisa establecida en la convención de Naciones Unidas de 1988 contra el tráfico ilícito de narcóticos y sustancias psicotrópicas, así mismo, los compromisos establecidos ante la OACI, para garantizar la seguridad de la operación aérea por parte de los estados contratantes.

El programa $\mathrm{ABD}$, es un modelo exitoso en lo referente a interdicción, y no solamente por el hecho de cumplir con los procedimientos establecidos, sino por el respeto a los DDHH, y a la reducción del tráfico aéreo ilegal, pero este resultado no obedece solamente al programa en sí, sino a una serie de iniciativas nacidas en la Dirección de Defensa Aérea de la Fuerza Aérea Colombiana, gestadas desde la perspectiva de la metodología seis sigmas.

Bajo el enfoque de seis sigma ${ }^{5}$ en el año 2005 se definió el problema como:

"En el proceso ABD, la reacción a trazas sospechosas es punto de medición para el indicador de efectividad de la Fuerza. Este valor se mide bajo el índice $\%$ de trazas sospechosas inutilizadas o inmovilizadas y se calcula como: (trazas inmovilizadas + trazas inutilizadas) / Total trazas sospechosas. De acuerdo a la RAE el valor ideal de este índice es de 85\%. Para el año 2003 el índice estaba en promedio en $1.89 \%$ basado en un total de 639 Trazas Sospechosas y una efectividad de solo 28 resultados exitosos y el impacto que generó es el transporte de 1 tonelada por vuelo

Metodología de optimización de procesos inventada por Motorola en los años 80 . 
a 25.000 dólares cada tonelada con unos ingresos promedio de U\$15.975.000.000 anuales, para los grupos al margen de la ley.” (Quintero, 2009b).

Para ese año el proceso se encontraba en -0,58 Sigma, lo que significa que se tenía la oportunidad de mejorar el proceso ya que cada millón de veces que se ejecutaba el mismo solamente se acertaba en 18.900 veces, en términos porcentuales representaba que el $98 \%$ de las veces se fallaba en la interdicción aérea, por lo tanto fue mandatorio tomar medidas para lograr mejorar el proceso y lograr reducir este movimiento.

Partiendo de este escenario se establecieron varias líneas de acción, donde se efectuó un planeamiento y estableció una estrategia de cómo abordar el problema.

Se inicia con el cruce de información existente y a establecer qué datos no se poseían, como la ubicación de pistas ilegales, matrículas de aeronaves, empresas, funcionarios de aviación, rutas principales, cultivos ilícitos, zonas de producción, organizaciones involucradas, y de qué manera se podía atacar este flagelo, con lo que se definió una hoja de ruta para aunar esfuerzos y lograr el objetivo de garantizar la seguridad del espacio aéreo nacional.

En primera instancia, se inician los trabajos para tomar las acciones necesarias a nivel nacional, involucrando a las agencias competentes que podían ayudar en la interdicción y es como se logra establecer acuerdos de servicio con entidades como la Policía Antinarcóticos, Grupo Control de Aviación Civil, Fiscalía General de la Nación, UAEAC, en razón a que la estrategia nacional para la lucha contra las drogas define diferentes roles, y la Fuerza Aérea Colombiana, necesitaba articular los esfuerzos para obtener un resultado contundente.

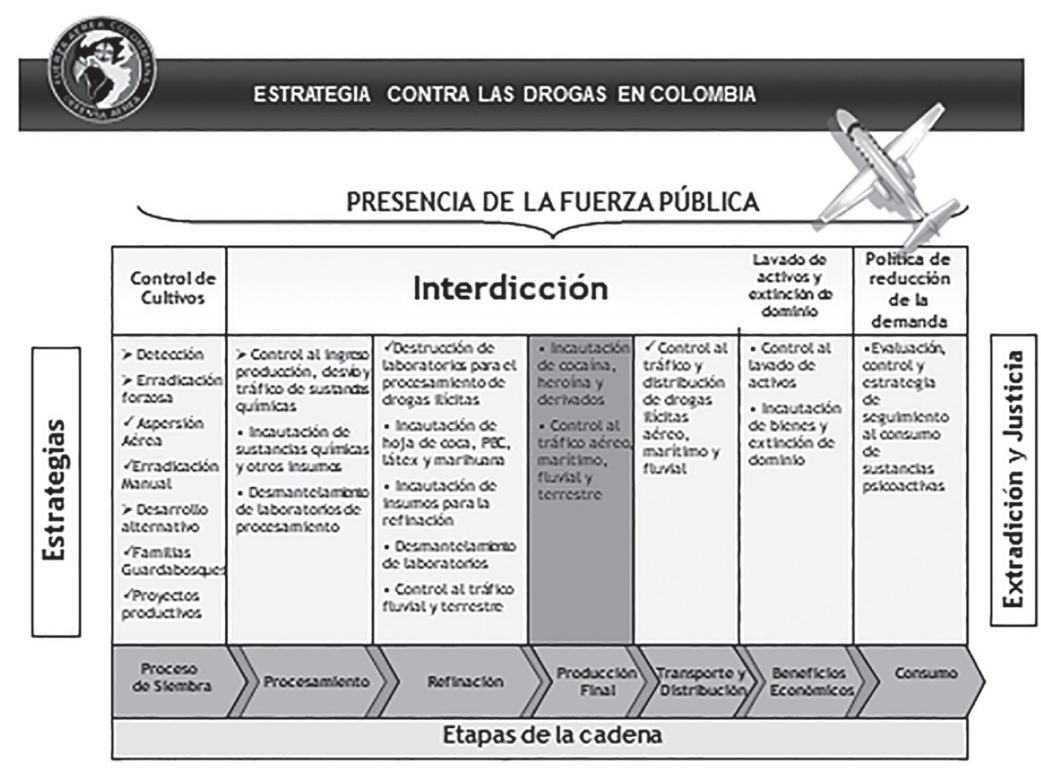

Fuente: Dirección de Defensa Aérea FAC(Quintero, 2013) 
Como se puede observar en dicha estrategia la Fuerza Aérea solamente estaba inmersa en la etapa de producción final y Transporte y distribución, pero la cantidad de actores que intervienen en dicho negocio hizo necesario ampliar el espectro y establecer varias actividades orientadas a controlar el problema raíz.

Las aeronaves por su naturaleza requieren de todo el soporte en tierra, talleres, mantenimiento, cargue, despegue, aterrizaje, personal entrenado, descargue, licencias, permisos, planes de vuelo, información meteorológica, tripulantes, etc, por lo tanto, parte del esfuerzo se focalizó en estos temas y se definieron cuáles eran las principales actividades en las cuales se debía enfocar el esfuerzo.

Fue así, como se priorizaron las rutas, pistas ilegales y aeronaves, estableciendo patrones de conducta que permitieron incrementar la efectividad de la interdicción, con lo que se dieron los primeros pasos hacia una estrategia que garantizara, el control del espacio aéreo, para llegar inicialmente a lograr el control del espacio aéreo nacional y posteriormente empezar a establecer alianzas, no solamente con países limítrofes sino con países con fronteras no adyacentes.

De esta manera es, como la Fuerza Aérea definió el Blanco Aéreo: "Son todos aquellos medios aéreos y su infraestructura de apoyo, que en alguna medida son empleados por el enemigo para controlar su espacio aéreo o que utiliza para vulnerar el nuestro. Siendo así, se puede determinar cómo componentes del blanco aéreo los siguientes: Aviones, Pilotos y Personal Aeronáutico, Pistas, Rutas, Infraestructura aeronáutica, Empresas, Espectro electromagnético, Sistemas de Comando y control" (Quintero, 2009), de igual manera se define una estrategia puntual sobre el tema: "Desarrollar las actividades necesarias para obtener el conocimiento del enemigo aéreo, con el fin de descifrarlo en su funcionamiento como en su ubicación, a fin de proceder a su neutralización física o legal mediante la realización de alianzas estratégicas interinstitucionales e internacionales"(QUINTERO, 2009), dándole vida a la integración con países tanto limítrofes geográficamente como con fronteras no adyacentes, al perseguir el mismo fin.

Igualmente, se define como objetivo principal "Minimizar, controlar o neutralizar la amenaza aérea actual o inminente, mediante el empleo de los medios de la Defensa Aérea" (Buelvas et al., 2016) (Quintero, 2009a), donde se deja en claro la problemática para el país y por ende la decisión del estado de enfrentar dicha amenaza a través de las siguientes acciones:

1. "Conocimiento claro de la amenaza.

2. Alianzas Estratégicas Interinstitucionales.

3. Fortalecimiento Normativo.

4. Control de ejes del enemigo.'(Quintero, 2009)

Partiendo de este enfoque, se inicia la búsqueda de información en varios frentes, en lo referente al primer punto se definieron las siguientes actividades: 
antecedentes, análisis de movimientos aéreos, análisis de movimientos legales, información de otras agencias, proyectos y blancos de defensa aérea, análisis operacional, gestión operacional, control de pistas ilegales. (QUINTERO, 2009).

En segundo término, se establecieron alianzas interinstitucionales con: Dirección Nacional de Estupefacientes, Policía antinarcóticos, Fiscalía General de la Nación, Armada Nacional de Colombia, Ejército Nacional, Departamento Administrativo de Seguridad, y Acuerdos y convenios de cooperación transnacional con: DEA, ICE,NAS,JIATF-South, por parte de EEUU con el convenio ABD (2003), y con Brasil (1997), Perú(2004), República Dominicana (2010), Venezuela(2011), Honduras (2011), Panamá (2012), Guatemala (2010) y Ecuador (2013), donde se definían procedimientos de cooperación para aunar esfuerzos en la lucha contra el delito transnacional aéreo.

En el tercer aspecto, se trabajó sobre la alineación de los procedimientos bajo la normatividad de DDHH, y se transversalizó sobre los definidos en los de operación con otros países estableciéndose controles administrativos y operacionales.

Finalmente, en el control de los ejes del enemigo se fortaleció la interdicción aérea, se aumentó el control sobre los campos de operación, se establecieron controles sobre empresas, aeronaves y personal.

Esta estrategia fue efectiva al lograr la integración de diferentes dependencias, no solo al interior de la Fuerza Aérea Colombiana, sino del país, y aún más allá, al involucrar a las fuerzas aéreas de otros países, con quienes se lograron estandarizar procedimientos y establecer canales de comunicación que permitían la sinergia entre estados limítrofes no adyacentes, logrando impactar el movimiento aéreo ilegal, se resume en el siguiente gráfico:

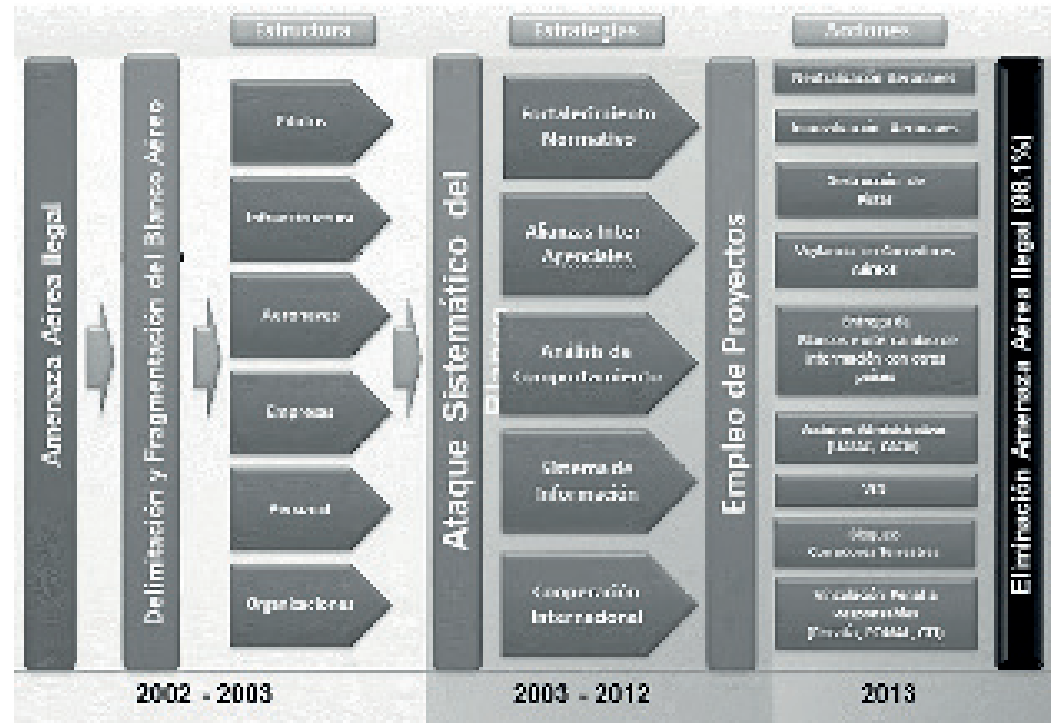

Fuente: Dirección de Defensa Aérea FAC(Quintero, 2013) 
Todos estos aspectos se tuvieron en cuenta en los procedimientos de control definidos con los países que establecieron acuerdos, lo que permitió aunar esfuerzos y ser mucho más eficientes en los resultados, donde no solamente se estandarizaron procedimientos, sino que se logró transferir conocimientos, experiencia y ayuda en la manera de efectuar el levantamiento de la información, sistematizarla, analizarla, distribuirla y emplearla de manera efectiva, de tal suerte que sirviera no solamente para el desarrollo operacional sino para allegar como elementos probatorios ante las autoridades judiciales y sirviera para consolidar casos que permitieran contrarrestar el actuar de los diferentes carteles, de esta forma se logró una reducción de los movimientos aéreos ilegales del espacio aéreo nacional, logrando una reducción del 96.8\% del año 2003 al 2013.

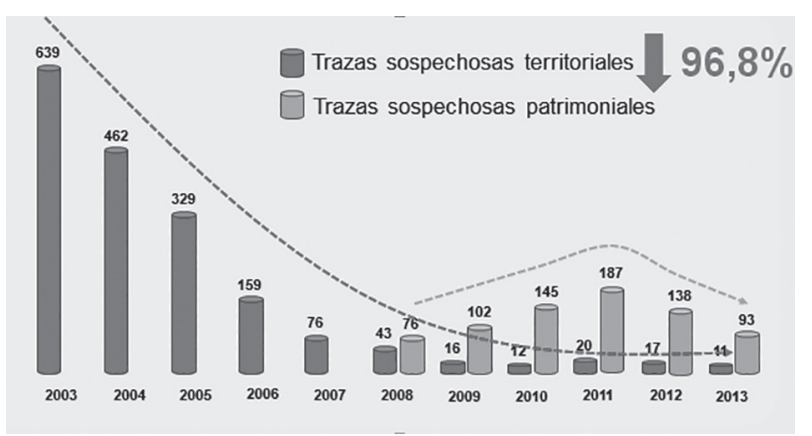

Fuente: Dirección de Defensa Aérea FAC(Quintero, 2013)

Para el 2008 el proceso se encontraba en 1.37 sigma lo que refleja una mejora significativa, en razón a que se logró una efectividad del $45 \%$ y a partir de este momento el proceso se estabiliza con unas reducciones mínimas, que no han podido ser mejoradas por la falta de aeronaves, radares, plataformas de alerta temprana y bases de lanzamiento, ya que estas medidas requieren inversiones cuantiosas de las que se tienen los proyectos de inversión pero no la asignación de recursos, sin embargo el panorama de sobrevuelo de aeronaves ilegales por el espacio aéreo nacional es completamente diferente a la del año 2003. 


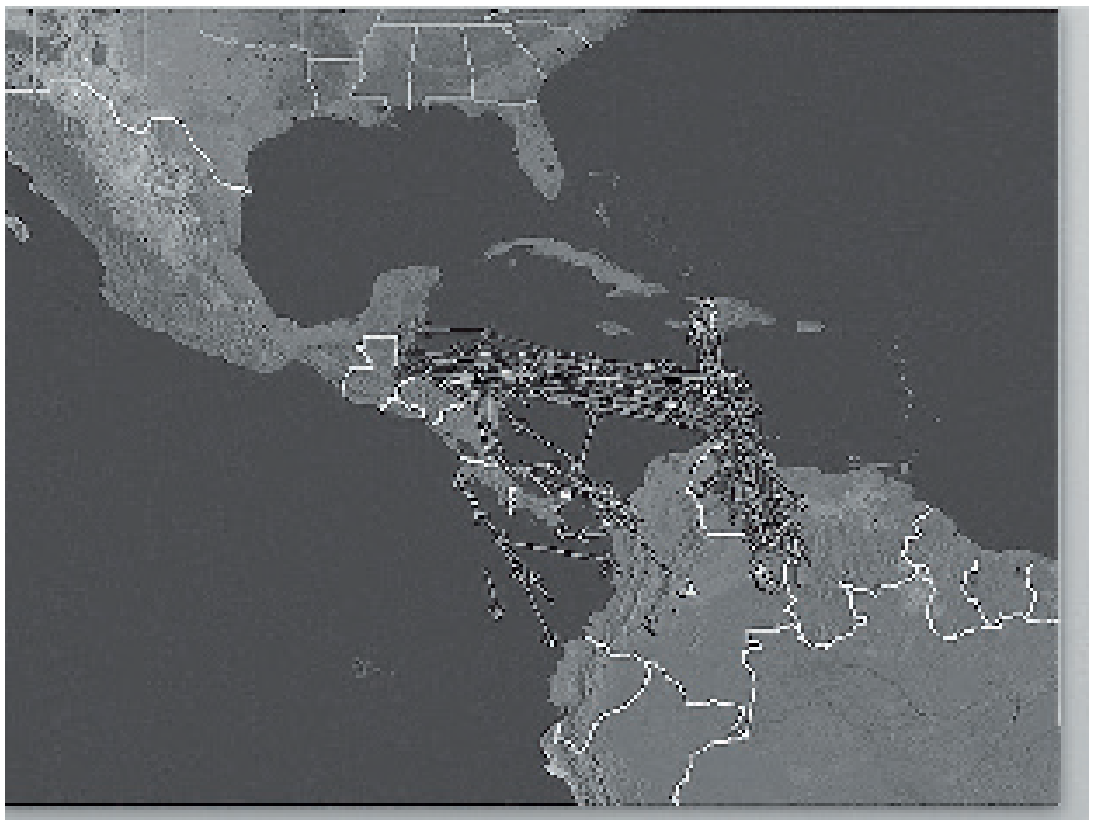

Fuente: Dirección de Defensa Aérea FAC (Quintero, 2013)

Como puede observarse esta estrategia ha sido efectiva desde la perspectiva aérea, sin embargo el fenómeno de los movimientos ilegales migró del ámbito aéreo al marítimo por varias razones, en primer término debido a que los movimientos marítimos permiten mover mayores cantidades de alcaloides, en segundo término las instalaciones requeridas como puerto para el zarpe de las embarcaciones no requieren mayores exigencias técnicas y dificultan enormemente su ubicación, en tercera instancia los medios técnicos de detección de estos movimientos son mínimos frente a la amenaza y su ubicación en tierra limita el alcance de los mismos y los instalados a bordo de aeronaves no permiten mantener una vigilancia de los mares de manera permanente, lo que dificulta su interdicción, así mismo el tiempo de permanencia de dichas embarcaciones es mucho mayor donde dos de los principales enemigos de las autoridades es el tiempo y el alcance, si no se tienen los medios suficientes para lograr transferir los blancos de un país a otro se pierden los esfuerzos y en segundo término si no se poseen las unidades capaces de llegar hasta las rutas que estas embarcaciones usan se hace imposible su interdicción, y no todos los países de la región poseen dicha capacidad, a excepción de EEUU, México y Colombia, quienes no alcanzan a cubrir las necesidades de vigilancia de estos corredores, lo que ha permitido el incremento de movimientos marítimos, su evolución tecnológica, su capacidad de carga y su alcance, tema que amerita un estudio aparte por sí mismo. 


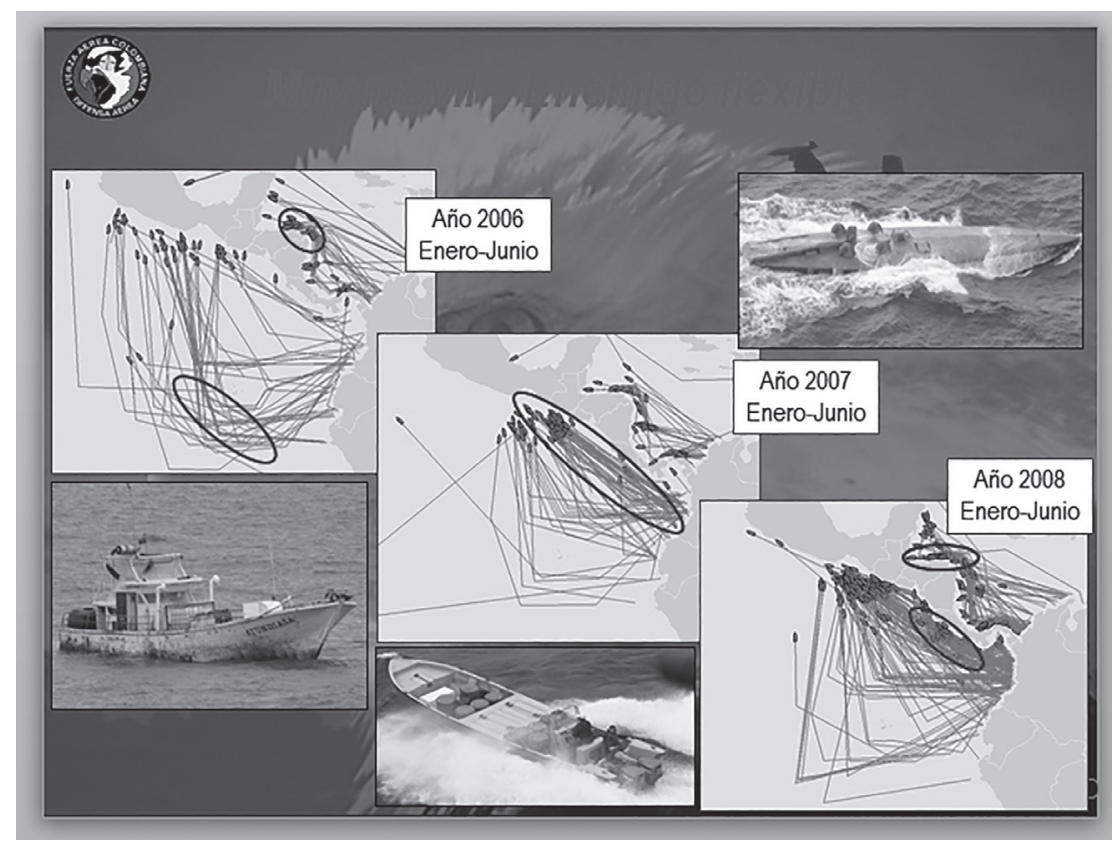

Fuente: Dirección de Defensa Aérea FAC(Quintero, 2009)

Del ejemplo anterior, se demuestra que los países no adyacentes pueden ser limítrofes y llegar a compartir varios intereses sobre un mismo tema, convirtiéndolos en países limítrofes en lo referente a estos intereses, de la misma manera se pueden plantear intereses de diferente índole, pero se va a encontrar siempre la misma situación, la unión de actividades orientadas a alcanzar el mismo interés, como se puede ver en los acuerdos comerciales, judiciales, aduaneros, migratorios o inclusive coaliciones con fines de efectuar operaciones militares, a nivel táctico se convierten en países limítrofes aunque sus jurisdicciones geográficas no se toquen.

Hoy en día con el advenimiento de no solamente rutas comerciales más cortas y directas, se establecen nuevos límites entre los Estados sino que se dan nuevas relaciones que van más allá de la capacidad estatal, cuando por ejemplo hay adeptos del DAESH alrededor del mundo, con lo cual se puede configurar lo que en la práctica podría llamarse un Estado Virtual, cuya influencia llega hasta donde tiene sus seguidores y hace de estos, países con fronteras no adyacentes, de manera similar se puede observar grupos como los que apoyan la independencia de diferentes estados, que de acuerdo a la ONU son 16 territorios en el mundo que aún no consiguen su independencia, y que Naciones Unidas define como territorios no autónomos, sin embargo se encuentran casos en Europa que por el momento no hacen parte de este inventario, como Gibraltar, Groenlandia, Irlanda 
del norte, Cataluña, El país Vasco, Escocia, Baviera, Flandes, Galicia, Islas Feroe, Padania, Tirol del sur, Córcega y Trentino,) por otra parte en Asia se encuentra el Tíbet, el Kurdistán, Hong Kong, Guam, Chechenia, Macao, Cachemira, Transnitria y Gagauzia, , así mismo en África se encuentra Somalilandia, Nueva Caledonia, Sahara Occidental y Tokelau, en América Bermudas, Islas Caimán, Islas Malvinas, Islas Turcas y Caicos, región Mapuche, islas Falklands, Quebec (el tiempo) y la lista continúa, así mismo los adeptos y detractores existen en todas partes y por diferentes motivos, que van desde lo económico, político, religioso, étnico, cultural, geopolítico, a continuación se puede ver la lista que Naciones Unidas tiene como territorios no autónomos, como puede verse con un sesgo político ya que no incluye una gran cantidad de territorios alrededor del mundo.

\begin{tabular}{|c|c|c|c|c|}
\hline Territorio & Incluidos en la lista & Administración & $\begin{array}{c}\text { Superficie } \\
(\mathrm{km} 2)^{1}\end{array}$ & Población 1 \\
\hline \multicolumn{5}{|l|}{ ÁFRICA } \\
\hline Sảhara Occidental 図面 & Descle 1963 & $\underline{2}$ & 266,000 & 584,000 \\
\hline \multicolumn{5}{|l|}{ ATLÁNTICO Y EL CARIBE } \\
\hline Anguila 저메 & Desde 1946 & Reino Unido & 96 & 15,700 \\
\hline Bermuda 図四 & Desde 1946 & Reino Unido & 53.35 & 61,695 \\
\hline 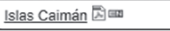 & Desde 1946 & Reino Unido & 264 & 60,413 \\
\hline 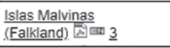 & Desde 1946 & Reino Unido & 12,173 & 2,500 \\
\hline $\begin{array}{l}\text { Islas Turcas y } \\
\text { Caicos 젖aㅔ }\end{array}$ & Desde 1946 & Reino Unido & 948.2 & 37,910 \\
\hline 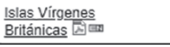 & Desde 1946 & Reino Unido & 153 & 28,200 \\
\hline $\begin{array}{l}\text { Islas Virgenes de los } \\
\text { Estados Unidos 젓 }\end{array}$ & Desde 1946 & Estados Unidos & 352 & 103,700 \\
\hline Monserrat 四四 & Desde 1946 & Reino Unido & 103 & 5,000 \\
\hline Santa Elena & Desde 1946 & Reino Unido & 310 & 5,691 \\
\hline \multicolumn{5}{|l|}{ EUROPA } \\
\hline Gibraltar & Descle 1946 & Reino Unido & 5.8 & 33,140 \\
\hline \multicolumn{5}{|l|}{ PACIFICO } \\
\hline Guam 중.m & Desde 1946 & Estados Unidos & 540 & 159,358 \\
\hline Nueva Caledonia & $1946-1947$ y desde 1986 & Francia & 18,575 & 268,767 \\
\hline Pitcaim 중 & Desde 1946 & Reino Unido & 35.5 & 39 \\
\hline Polinesia Francesa 펑미 & 1946-1947 y desde 2013 & Francia & 3,600 & 271,800 \\
\hline Samoa Americana 저시 & Desde 1946 & Estados Unidos & 200 & 60.200 \\
\hline Tokelau & Desde 1946 & Nueva Zelandia & 12.2 & 1,499 \\
\hline
\end{tabular}

Fuente: Las Naciones Unidas y la Descolonización. http://www.un.org/es/decolonization/ nonselfgovterritories.shtml

Como puede verse cada una de estas causas tiene adeptos y detractores que no solamente sientan sus puntos de vista desde lo ideológico, sino que cubre aspectos publicitarios, hasta actividades en las redes sociales que buscan apoyo alredor del mundo, pero así mismo encuentran detractores, todo esto en detrimento de los límites geográficos de los estados comprometidos y donde terceros países con intereses específicos apoyan y facilitan dichas actividades, mientras que contrapartes bloquean y desmotivan estos procesos y cada caso tiene ejemplos, inclusive podría hacerse referencia a los casos más relevantes de los últimos años, como el Brexit, donde siempre existió la duda sobre la intervención de Rusia en dicha consulta, o las elecciones presidenciales de los EEUU, donde nuevamente 
Rusia deja un manto de duda sobre su intervención en las mismas, nuevamente permitiendo ver que las fronteras no adyacentes son una realidad en la actualidad y que en lo que se puede preveer va a terminar cobrando una mayor relevancia, debido a que los intereses de los Estados van más allá de sus fronteras que serán cambiantes pero siempre existirán.

\section{REFERENCIAS}

¿En qué regiones europeas quieren la independencia?|Europa |DW |29.09.2017. (n.d.). Retrieved March 19, 2018, from http://www.dw.com/es/en-qué-regiones-europeas-quieren-la-independencia/a-40751717

Buelvas, E. P., Gehring, H., Eduardo, E., Buelvas, P., Mauricio, A., Camilo, V., ... García, R. (2016). Política exterior colombiana: escenarios y desafíos en el posconflicto. Retrieved from https://s3.amazonaws.com/academia.edu. documents/46571501/la politica exterios colombiana en el marco del posconflicto.pdf?AWSAccessKeyId=AKIĀIWOWYY $\overline{\mathrm{G} Z} \overline{2} \overline{\mathrm{Y}} \overline{\mathrm{B}} \mathrm{UL} 3 \overline{\mathrm{A}} \& \overline{\mathrm{Ex}}$ pires $=1512315720 \&$ Signature $=\mathrm{CU} \% 2 \mathrm{BtsDX3nCd1NGxYnOG65 \textrm {cErDqg }}$ $\% 3 \mathrm{D} \&$ response-content-disposition=inline

DANE. (2003). comunicado dane 4 trim 2003.

Devia, H. R., Iván, D., \& Ferreira Cardenas, M. (2015). Modelamiento estadístico del tráfico aéreo ilegal en Colombia.

FERNÁNDEZ-CARrión, M.-H. (2010). Historiografía, metodología y tipología de fronteras.

Foucher, Michael. (1946-... . ). A. du texte. (1987). L'invention des frontières. Retrieved from http://gallica.bnf.fr/ark:/12148/bpt6k3322804w

González, L., \& Villasenín, Y. (2010). Cocaína. Udc.es, 21.

HARTSHORne, R. (1939). The nature of geography. Retrieved from https:// ia800309.us.archive.org/31/items/natureofgeograph010994mbp/natureofgeograph010994mbp.pdf

JimÉNEZ, A. (1994). Handbook of Hispanic cultures in the United States.

KaUtiLyA. (2008). Arthasastra La ciencia politica de la adquisición y el mantenimiento de la tierra. (O. Guerrero, Ed.) (Universida). México.

LóPEZ, R. D. (1981). El mar territorial y el patrimonial. Retrieved from file://C:/ Users/Henry/Downloads/5422-10995-1-SM.pdf

Quintero, H. (2009a). Cumbre de presidentes Colombia. México: Guatemala y Panamá. 
Quintero, H. (2009b). Reunión jefes de operaciones.

Quintero, H. (2013). Cómo se controló el blanco aeronáutico.

Ranking de Estados que aspiran a su independencia-Listas en 20minutos.es. (n.d.). Retrieved March 19, 2018, from https://listas.20minutos.es/lista/ estados-que-aspiran-a-su-independencia-347871/

Según la ONU, en el mundo todavía hay 16 territorios no autónomos colonias que no pueden ser países-Archivo Digital de Noticias de Colombia y el Mundo desde 1990-eltiempo.com. (n.d.). Retrieved March 19, 2018, from http://www.eltiempo.com/archivo/documento/MAM-4017672

TURNER, F. J. (1893). El significado de la frontera en la historia americana. 